\title{
COMPARACIÓN ENTRE LOS PROMEDIOS DE LA PRUEBA DE APTITUD ACADÉMICA Y LA PRUEBA DE HABILIDADES CUANTITATIVAS DE LOS ESTUDIANTES DE LA UNIVERSIDAD DE COSTA RICA
}

\section{COMPARISON BETWEEN THE AVERAGE OF ACADEMIC APTITUDE TEST AND QUANTITATIVE SKILLS TEST OF STUDENTS AT THE UNIVERSITY OF COSTA RICA}

\author{
Marianella Bolaños Barquero* \\ Luis Rojas Torres ${ }^{* *}$
}

\begin{abstract}
RESUMEN
En este artículo se presentan los resultados que se obtuvieron con la aplicación de la Prueba de Habilidades Cuantitativas durante el primer semestre del 2010, a 206 estudiantes de primer ingreso de la Universidad de Costa Rica (UCR), matriculados en las carreras de Física, Estadística y Matemática. En el análisis se procedió a comparar los promedios obtenidos en la Prueba de Habilidades Cuantitativas con los resultados obtenidos en la Prueba de Aptitud Académica, así como, el rendimiento académico obtenido en los cursos introductorios y del tercer ciclo de esas carreras. Para esto se utilizó la técnica estadística del Análisis de Variancia (ANova) y la prueba de comparaciones múltiples de Tuckey.
\end{abstract}

PALABRAS CLAVE: UNIVERSIDAD * PRUEBA DE APTITUD * HABILIDAD * RENDIMIENTO ACADÉMICO

\section{ABSTRACT}

This article presents the results obtained with the application of Quantitative Skills Test during the first semester of 2010, to 206 first year students at University of Costa Rica (UCR), registered in the careers of Physics, Statistics and Mathematics. In this analysis the means obtained from the Quantitative Skills Test were compared with the results from the Academic Aptitude Test, as well as with the academic performance obtained in introductory courses and the third cycle of those careers. This project was done by using both, the statistical technique of Analysis of Variance (ANOVA) and the Turkey multiple comparisons test.

\section{KEYWORDS: UNIVERSITIES * APTITUDE TEST * SKILLS * ACADEMIC PERFORMANCE}

* Instituto de Investigaciones Psicológicas de la Universidad de Costa Rica (UCR) y Escuela de Matemática de la Universidad Nacional de Costa Rica (UNA).

marianela.bolanos@UcR.ac.cr

** Instituto de Investigaciones Psicológicas y Escuela de Matemática, ambos de la Universidad de Costa Rica (UCR). luismiguel.rojas@UcR.ac.cr 


\section{INTRODUCCIÓN}

La educación es una herramienta esencial para la población porque promueve el desarrollo humano, le permite a las personas insertarse a la sociedad y junto a la adquisición de una formación superior por parte de esta, proporciona también una mayor movilidad social y por lo tanto, la posibilidad de una mejor calidad de vida; de ahí que la demanda por una educación superior ha venido en aumento en las últimas décadas en todo el mundo (Escrigas $y$ Lobera, 2009). En Costa Rica, según el Tercer Informe Estado de la Educación del Programa Estado de la Nación (2011), un 6,4\% de la población reportó que asistía a la educación universitaria en el 2009 (1,2 puntos porcentuales más que a inicios de esta década), período en el cual la matrícula total de estudiantes en las cuatro universidades públicas creció un $36,1 \%$.

Ante este aumento en la demanda, las universidades estatales se ven en la necesidad de implementar procesos de selección. En el caso de la Universidad de Costa Rica (UCR), el proceso de selección se hace con base en el promedio de admisión obtenido por los estudiantes que optan por ingresar a esta institución. Dicho promedio está compuesto por un indicador de las notas obtenidas en los últimos dos años de secundaria y por la calificación obtenida en una prueba elaborada por la ucR, denominada Prueba de Aptitud Académica (PAA). Cabe resaltar que ambos componentes a lo largo de los años han correlacionado significativamente con el éxito académico de los estudiantes de la Universidad $y$ de ahí la justificación de por qué son tomados en cuenta para los procesos de selección (Rojas, 2012; Jiménez y Morales, 2010).

En la ucr son pocas las Unidades Académicas que solicitan otros requisitos especiales para ingresar a determinada carrera, pese a que en los perfiles de ingreso se menciona una lista de habilidades y características deseables, necesarias para poder desenvolverse exitosamente en las diferentes áreas (ucR, Oficina de Orientación, 2010); por lo que el ingreso a la mayoría de las carreras depende únicamente del promedio de admisión, situación que deja de lado estas habilidades y se contrapone a lo expuesto por Tourón (1984) al afirmar que: ...a la Universidad deben acceder aquellas personas que realmente estén capacitadas para los estudios que en ella se ofrecen. Sería demagógico y equivocado, al tiempo que un engaño a los estudiantes, permitir el ingreso a la universidad a personas que no tienen las condiciones adecuadas, y que antes o después terminarán por abandonar sus estudios (1984: 23).

En cuanto a las aptitudes específicas, se ha señalado que determinadas carreras requieren unas aptitudes más desarrolladas que otras. Por ejemplo, las ingenierías precisarían de un buen dominio y desarrollo de la capacidad numérica, abstracta y de razonamiento espacial (González, 1989). En particular, en las carreras de Física, Matemática y Estadística pertenecientes a la Universidad de Costa Rica, los perfiles de entrada mencionan entre las habilidades y características deseables a las habilidades numéricas; sin embargo, actualmente en el sistema de admisión a estas carreras no se toma en cuenta la presencia de esta habilidad en los estudiantes que desean ser admitidos. Esta situación puede ser la causa del por qué en estas carreras hay índices tan altos de deserción y rezago, los cuales traen como consecuencia una tasa muy baja de graduación. Según la oficina de Planificación Universitaria de la ucR (2012), de 1998 a 2007, la cantidad de graduados en las carreras de Física, Matemática y Estadística fue de 97, 30 y 126 estudiantes respectivamente, lo cual indica que al año se gradúan en promedio: diez estudiantes en Físi$\mathrm{ca}$, tres estudiantes en Matemática y trece estudiantes en Estadística; lo cual es una cantidad muy pequeña en comparación con la cantidad de estudiantes admitidos a estas carreras.

La situación antes descrita reta a la Universidad a elaborar mecanismos de selección que además tomen en cuenta las habilidades mínimas deseables para poder ingresar a cada carrera. Ante esta situación surge el Proyecto de Habilidades Cuantitativas (нс), el cual pretende que "en aquellas carreras que requieren de destrezas en el campo matemático, logren mejorar el proceso de selección de sus estudiantes y garantizar de alguna forma la permanencia $y$ 
graduación de estos" (Proyecto de Habilidades Cuantitativas, 2010: 3). Basado en las afirmaciones anteriores surge este estudio, el cual tiene como finalidad comparar los promedios de la PAA $y$ la prueba de нс entre los estudiantes aprobados, avanzados, reprobados, rezagados y desertores, de las carreras de Física y Meteorología, Matemática Pura y Ciencias Actuariales, así como, los de Estadística.

\section{REFERENTES TEÓRICOS}

En este apartado se presentan aspectos teóricos referidos al rendimiento académico $y$ al razonamiento cuantitativo, elementos relevantes para este estudio. Se hace alusión a las pruebas específicas de ingreso a carrera, como una herramienta complementaria en los procesos de selección y se mencionan estudios que corroboran la relación entre el razonamiento cuantitativo y el rendimiento académico de los estudiantes que cursan las carreras de Física, Estadística y Matemática.

El rendimiento académico que ha sido motivo de estudio en muchas investigaciones, es hoy aún más significativo por la dinámica que experimenta el sector universitario, ante una sociedad que se caracteriza por el rápido avance del conocimiento, la fluidez en la transmisión de la información y los cambios acelerados en las estructuras sociales (Garbanzo, 2007).

En Jiménez (1994), el rendimiento académico se define como el nivel de conocimientos demostrado en un área o materia, comparado con la norma de edad y nivel académico. De Spinola (1990) lo define como el cumplimiento de las metas, logros u objetivos establecidos en el programa o asignatura que se encuentra cursando el estudiante.

Las definiciones anteriores justifican por qué en varias investigaciones, los indicadores más utilizados para el rendimiento académico son las calificaciones de los estudiantes. Las notas (indicador de la certificación de logros) parece ser el mejor indicador o al menos, el más accesible para definir el rendimiento académico (Boza, 1970; González, 1990 y Pérez, 1991). Por su parte, Montero, Villalobos y Valverde (2007) expresan que las calificaciones constituyen en sí mismas el criterio social y legal del rendimiento académico de un estudiante en el ámbito institucional. La forma más directa de establecerlas es por medio de exámenes o pruebas de medición. Page, Moreal, Calleja, Cerdan, Echevarria, García, Gaviria, Gómez, Jiménez, López, Martín-Javato, Mínguez, Sánchez y Trillo (1990) manifiestan que los indicadores más utilizados para el rendimiento académico han sido las calificaciones $y$ las pruebas objetivas o tests de rendimiento creados ad hoc (1990: 24).

Pérez, Ramón, Sánchez (2000) y Vélez, Roa (2005) confirman lo anterior, cuando expresan que el rendimiento académico se mide mediante las calificaciones obtenidas, con una valoración cuantitativa, cuyos resultados muestran las materias ganadas o perdidas, la deserción y el grado de éxito académico.

Garbanzo (2007) define el rendimiento académico como "la suma de diferentes y complejos factores que actúan en la persona que aprende $y$ ha sido definido con un valor atribuido al logro del estudiante en las tareas académicas" (2007: 46). Esta definición lleva implícito que el rendimiento académico se ve afectado por una diversa cantidad de factores, los cuales deben ser tomados en consideración en el momento de hacer investigaciones sobre el rendimiento académico. Page et ál. (1990) corroboran esta situación cuando exponen que los factores que influyen en el rendimiento académico de los alumnos, también llamados "determinantes" del rendimiento académico, son difíciles de identificar, "pues dichos factores o variables conforman muchas veces una tupida maraña, una red tan fuertemente entretejida, que resulta ardua la tarea de acotarlas o delimitarlas para atribuir efectos claramente discernibles a cada uno de ellos" (1990: 29).

En las investigaciones sobre rendimiento académico universitario se consideran gran cantidad de factores de tipo cognitivos, emocionales y socioeconómicos, además de los resultados en la educación media, pruebas nacionales, exámenes de ingreso a universidades, pruebas de aptitudes intelectuales, entre otros. Montero et ál. (2007) consideran entre las variables asociadas al rendimiento académico: los factores institucionales, pedagógicos, psicosociales y 
sociodemográficos. Para González (1990 y 1993), los factores que mejor predicen el rendimiento académico universitario son las variables biográficas, el rendimiento académico previo, la inteligencia, las aptitudes $y$ funciones mentales, los rasgos de personalidad $y$ las variables motivacionales. Por su parte, Garbanzo (2007) considera que entre los determinantes personales asociados al rendimiento académico se encuentran la competencia cognitiva, la inteligencia y las aptitudes. También, considera los determinantes sociales y los determinantes institucionales, incluyendo en este último las pruebas específicas de ingreso a la carrera, pruebas que según Cascón (2000), tienen el potencial de detectar posibles grupos de riesgo de fracaso escolar; al igual que para Russell, Persing, Dunn y Rankin (1990), para quienes la medición cognitiva es un aspecto clave para predecir éxito en ambientes educacionales universitarios.

Con respecto a estas pruebas, Thurstone, L. y Thurstone, T. (1941) y Guilford (1967) afirman que se deberían dejar de usar pruebas generales de inteligencia $y$ que es mejor medir cada factor por separado y obtener un perfil de las habilidades de una persona, lo que da más información para orientación vocacional o para planear su educación, lo cual indica la relevancia de las pruebas específicas en la predicción del rendimiento académico. Edel afirma que "en la vida académica, habilidad y esfuerzo no son sinónimos, el esfuerzo no garantiza un éxito, $y$ la habilidad empieza cobrar mayor importancia" (2003: 1).

Las habilidades han sido motivo de estudio durante mucho tiempo, en particular, las habilidades intelectuales, lo que ha permitido la generación de diversas teorías, entre ellas la de Carroll (1993); en la que se plantea la estructura de la inteligencia denominada Teoría de los Tres Estratos. En esta se analizan cientos de bases de datos mediante el análisis factorial exploratorio y se concluye con la caracterización de los factores encontrados. Luego, se realiza un análisis factorial exploratorio con los factores hallados en la primera etapa, encontrándose que estos factores se aglomeran en tres grupos linealmente independientes, los cuales se denominaron: razonamiento inductivo, razonamiento general secuencial y razonamiento cuantitativo. Los factores que cargan altamente en la dimensión razonamiento cuantitativo son medidos por tests que llevan nombres como: Razonamiento Aritmético, Aptitud Matemática, Aritmética, etc.

Thompson (1993) define razonamiento cuantitativo como el análisis de una situación dentro de una estructura cuantitativa (red de relaciones entre cantidades). Lobato y Siebert (2002) mencionan que el razonamiento cuantitativo implica concebir una situación dada en términos de cantidades mediante la construcción de redes de relaciones cuantitativas. Karim (s. f.) expresa que es el proceso de explorar, mostrar, juzgar y llegar a conclusiones a partir de información cuantitativa y para Steen (2004), el razonamiento cuantitativo es un "razonamiento sofisticado con las matemáticas elementales en lugar de un razonamiento elemental con las matemáticas sofisticadas" (2004: 9).

Según el Consejo Nacional de Profesores de Matemática de los Estados Unidos (National Council of Teachers of Mathematics-мстм, 2000), el razonamiento cuantitativo es la habilidad desarrollada para poder analizar la información cuantitativa y para determinar cuáles son las habilidades $y$ procedimientos que pueden aplicarse para llegar a la solución de un problema que contenga este tipo de información. El кстм menciona que la habilidad cuantitativa tiene como foco la resolución de problemas, por lo cual para este razonamiento, se hacen necesarias las siguientes seis capacidades:

1) Lectura y comprensión de información en varios formatos.

2) Interpretación de la información cuantitativa y realización de inferencia a través de ella.

3) Resolución de problemas usando métodos aritméticos, algebraicos, geométricos o estadísticos.

4) Estimación de respuestas y comprobación de su veracidad.

5) Comunicar la información cuantitativa.

6) Reconocer las limitaciones de los métodos matemáticos y estadísticos. 
Estas capacidades permiten ejemplificar aún más que la habilidad cuantitativa no es conocimiento matemático, sino la habilidad asociada a la comprensión de la información cuantitativa (Dwyer, Gallagher, Levin y Morley, 2003). Siguiendo a estos autores, el razonamiento cuantitativo es "la capacidad de analizar la información cuantitativa, incluyendo la determinación de qué técnicas $y$ procedimientos se pueden aplicar a un problema particular para llegar a su solución" (2003: 12); ellos hacen énfasis en que el razonamiento cuantitativo no se refiere a las habilidades de cálculo, sino a la capacidad para determinar cuál es la mejor estrategia de cálculo para resolver un problema $y$ a la habilidad de inferir el grado de precisión requerido para estos cálculos. Agregan también que "el razonamiento cuantitativo requiere el uso de conocimientos matemáticos para propósitos de evaluación y generalmente más, para la resolución de problemas. Sin embargo el razonamiento cuantitativo es fundamentalmente diferente, en lo conceptual y en lo práctico, del conocimiento del contenido matemático" (2003: 2).

Por lo tanto, se infiere que en la elaboración de los test de razonamiento cuantitativo, en los cuales se requiere razonamiento basado en relaciones y propiedades matemáticas, que comúnmente presentan una variedad de problemas de razonamiento matemático, series de números y problemas que requieren la selección de una operación matemática apropiada, se debe considerar y controlar también, la cantidad de computación numérica, la carga de vocabulario, los componentes de visualización espacial y la complejidad numérica de las operaciones (De Juan-Espinosa, 1997), para no caer en la evaluación de otras dimensiones que pueden afectar la validez de la prueba. Se requiere también conocer de antemano la población sujeta a ser examinada, para que se constate que posee los conocimientos requeridos para poder llegar a las soluciones de los problemas planteados.

\subsection{RELACIÓN ENTRE EL RAZONAMIENTO} CUANTITATIVO Y EL RENDIMIENTO ACADÉMICO DE LOS ESTUDIANTES QUE CURSAN LAS CARRERAS DE FÍSICA, ESTADÍSTICA Y MATEMÁTICA

Estudios realizados confirman que existe relación entre el razonamiento cuantitativo de un individuo y su rendimiento académico en las carreras de Física, Estadística y Matemática. En el informe Graduate Record Examination (GRE) Predicción del logro de Doctorado en Psicología, Matemática y Química (1972), en el cual se trató de definir subgrupos para los que los exámenes GRE tuvieran diversos grados de exactitud de predicción y además, proporcionaran bio-perfiles gráficos de cada uno de estos subgrupos; se consideró para el área de Matemática una muestra de 845 , dividida en dos para que se pudiese realizar la validación cruzada; obteniéndose para ambas muestras correlaciones de 27 y .32 en el GRE-Verbal, $y$. 27 y .26 en el GRE-Cuantitativo. Por su parte, el GRe-Avanzado en la Prueba de Matemática, fue el mejor predictor individual con correlaciones de .38 y .44 para las dos muestras.

La correlación múltiple entre los cinco predictores (GRE-Verbal, Cuantitativo, Avanzado, Informes de referencia y Promedio de calificaciones de pregrado) y el logro del Ph. D. es bastante respetable (.40 en la muestra 1 y transversal válida a un 0.44 en la muestra 2), lo que indica una predicción relativamente precisa. En síntesis, fue más preciso en el área de postgrado de ciencias: Matemática y Química, que en Psicología. Cabe mencionar que el GRe posee una sección denominada Razonamiento Cuantitativo, que mide un constructo similar al de la prueba de Habilidades Cuantitativas de la ucr.

Schneider y Briel (1990) realizaron un estudio sobre la validez del GRE, cuyo propósito fue ayudar a las instituciones en la interpretación y uso de los resultados que brinda este test en el proceso de admisiones de postgrado, así como también medir la probabilidad de éxito de los estudiantes, mediante el Servicio de Estudios de Validez (Validity Study Service-vss) del GRE. En este estudio, los análisis se realizaron a dos grupos: estudiantes que se comunican mejor en inglés $y$ aquellos que se comunican mejor en algún otro idioma; las conclusiones se obtuvieron de 606 estudios de validez que se realizaron entre octubre de 1983 y setiembre de 1988, para los graduados de los distintos departamentos: Ciencias Naturales (donde se incluye Matemática y Física), Ingeniería, Ciencias Sociales, Humanidades y Artes, Educación y Negocios. Se obtuvo que los resultados de los 
exámenes generales (GRE General Test), tienden a mostrar correlaciones moderadas con el promedio del primer año y los resultados de los exámenes por materia específica (GRE Subject Test), incluso son mejores predictores del promedio del primer año que las puntuaciones generales. Pero la combinación de los resultados del GRE General Test y del promedio de calificaciones de bachillerato (Grade Point Average-GPA), se correlaciona más altamente con los promedios de primer año que los resultados del GRE O GPA por separado.

En resumen, los datos recolectados a través del vss $y$ analizados en este informe, indican que las pruebas GRE pueden ser parte útil del proceso de admisión. La combinación de los resultados del GRE General Test, Subject Test y el GPA tienen poder predictivo a pesar de ser factores que tienden a esconder el verdadero valor de las pruebas, como la restricción del rango y las prácticas de selección compensatoria, y aunque los predictores individualmente proporcionan información interesante, la combinación de estos es lo que se debe utilizar en la práctica. Sin embargo, estos resultados no sugieren que las puntuaciones del GRE $y$ GPA deben utilizarse como una fórmula para la admisión, más bien implica, que los resultados del GRE pueden ser especialmente importantes cuando un departamento hace esfuerzos por diversificar a sus estudiantes, al proveer el nivel de riesgo asociados a estos, ayudando así a la identificación temprana de aquellos que deben ser dirigidos $y$ apoyados para aumentar sus posibilidades de éxito.

En otra investigación realizada por Paglin y Rufolo (1990) sobre el capital humano heterogéneo, elección de la ocupación y diferencias de ingresos entre los hombres $y$ las mujeres, se muestra que los individuos tienen diferentes atributos iniciales que determinan una ventaja en la producción de diferentes tipos de recursos humanos; encontraron que la capacidad matemática es un factor determinante para la elección de un postgrado en los estudiantes universitarios, pues aquellos que obtuvieron un promedio alto en el área cuantitativa del GRE, se inclinan por carreras como Física, Bio-física, Astronomía, Matemática, Estadística, Ingenierías, entre otras del área científica-matemática. Agregan también que las diferencias en los salarios en los distintos campos de trabajo, se explica en gran medida, no por discriminación, sino porque las habilidades cuantitativas son más difíciles de hallar entre los individuos; encontrándose una alta correlación entre los atributos cuantitativos de un campo de trabajo y la media de los salarios iniciales de los graduados universitarios en ese campo.

Otro estudio realizado por Tirado, Backhoff, Larrazolo y Rosas (1997), en la Universidad Autónoma de Baja California, donde se utiliza desde 1992, el Examen de Habilidades y Conocimientos Básicos (Ехнсова) para seleccionar a sus nuevos estudiantes; la sección de Habilidades Cuantitativas resultó ser la parte de la prueba más importante en la predicción del rendimiento académico en el primer año, en las carreras del área económica-administrativas y la de física-matemáticas.

\section{METODOLOGÍA}

\subsection{INSTRUMENTO}

La prueba de Habilidades Cuantitativas se compone de cuarenta ítems de selección única, los cuales se agrupan en cuatro áreas de contenido: análisis de datos, aritmética, geometría y álgebra. La teoría implícita en estos ítems, aparte del razonamiento cuantitativo que se requiere, respeta los contenidos incluidos en el temario de Matemática hasta noveno año de la Educación General Básica costarricense; esto porque la población estudiantil que concluye este ciclo ha desarrollado un mínimo de contenidos, no así para el ciclo diversificado donde el currículo varía sustancialmente. El tiempo asignado para la resolución de esta prueba es de una hora cuarenta y cinco minutos, además no se permite el uso de la calculadora.

\subsection{MUESTRA}

La prueba de Habilidades Cuantitativas se aplicó en el primer semestre de 2010, a un total de 206 estudiantes de primer ingreso de la ucR, de los cuales 90 estaban matriculados en carreras de la Escuela de Física (Física y Meteorología), 65 en la carrera de Estadística y 51 en 
carreras de la Escuela de Matemática (Matemática Pura y Ciencias Actuariales).

La aplicación de la prueba fue realizada gracias al apoyo de las escuelas participantes, las cuales convocaron a todos los estudiantes de primer ingreso a carrera. Aunque la muestra no es estadísticamente representativa, se obtuvo un porcentaje de alcance mayor al $50 \%$ en todas las carreras.

\subsection{MÉTODO}

Inicialmente se deseaba realizar un estudio de predicción del rendimiento académico de estos estudiantes durante su primer año de estudios universitarios, lo cual resultó algo imposible de abarcar, debido a la pérdida de datos ocasionada por el alto porcentaje de rezago y deserción en las carreras estudiadas.

Ante esta situación se optó primeramente, por hacer una categorización de los estudiantes basados en los cursos introductorios, donde se establecen: a) aprobados, los que aprobaron el curso inicial de la carrera la primera vez que lo llevaron; b) reprobados, los que perdieron el curso inicial de la carrera al menos una vez y c) desertores, los que no llevaron el curso inicial de la carrera o se trasladaron de carrera.

Luego se procedió a comparar los promedios obtenidos en la prueba de Habilidades Cuantitativas (нс) entre cada uno de ellos, haciendo uso de la técnica estadística del Análisis de Variancia (anova) y de la prueba de comparaciones múltiples de Tuckey. Dichos análisis y la corroboración de sus supuestos se realizaron con el software R en su versión 2.15.1.

Además de realizar comparaciones entre los promedios obtenidos en Hc, también se realizaron comparaciones entre los promedios obtenidos en la Prueba de Admisión a la Universidad de Costa Rica (PAA), tomando en cuenta la sección de Matemática de la paA (ма-раA). Incluso se realizó otra categorización para la aplicación de las comparaciones múltiples.

Esta categorización está basada en los cursos de tercer ciclo y en ella se encuentran: a) avanzados, los estudiantes que cuatro ciclos después de ingresados a la carrera han aprobado al menos un curso del tercer ciclo; b) rezagados, los que llevaron el curso inicial de la carrera, pero cuatro ciclos después de ingresados a esta no han aprobado algún curso del tercer ciclo $y$ c) desertores, los que no llevaron el curso inicial de la carrera o se trasladaron de carrera.

Los cursos de carrera son aquellos que imparte la escuela de Física, Estadística y Matemática, de estos los primeros cursos son Física Introductoria (Fs-0227), Estadística Introductoria (xs-1110) y Principios de Matemática (ма0150). También se incorporó como curso inicial de Física, Algebra Lineal (ма-1004), debido a que por motivos de intenciones de traslado de carrera muchos estudiantes no llevan el curso Fs-0227, pero sí мA-1004.

En el tercer ciclo, los cursos de las carreras de Física son Laboratorio de Física General II (Fs-0311) y Física General para Físicos II (Fs0327). Para la carrera de Estadística, los cursos son Estadística Computacional I (xs-2210), Modelos Probabilísticos Discretos (xs-2310) y Métodos Estadísticos (xs-2110). Los que corresponden a la carrera de Matemática son Cálculo en una Variable II (ма-0350) y Álgebra Lineal (ма-0360). En Matemática, el curso Programación I (ci-1101) no le pertenece a la carrera, pero se consideró como tal, debido a la importancia que tiene en esta.

Finalmente, se aclara que la carrera de Ciencias Actuariales presenta el mismo curso introductorio que Matemática Pura y los mismos cursos de carrera en el tercer ciclo, igual situación ocurre con Meteorología y Física. Debido a esto se consideró Matemática Pura y Ciencias Actuariales como una sola población llamada Matemática, así como, a Física y Meteorología se le denominó simplemente Física.

\subsection{VARIABLES}

La medida utilizada para la Prueba de Habilidades Cuantitativas son los puntos obtenidos, los cuales varían de 0 a 40; para la PAA y sus secciones, la medida usada es el porcentaje de respuestas correctas, la cual oscila entre 0 y 100. Todos los cursos examinados se califican de 0 a 10 y se aprueban con una nota mínima de 7.0.

En la tabla 1 se presentan las estadísticas descriptivas obtenidas por la población examinada en las pruebas de interés, en la cual se puede apreciar que en las tres pruebas el promedio es cercano al $50 \%$ de aciertos. 
TABLA 1

ESTADÍSTICAS DESCRIPTIVAS DE LAS PRUEBAS UTILIZADAS

\begin{tabular}{lcccc}
\hline PRUEBA & MIN. & MEDIANA & MEDIA & MAX. \\
\hline PAA (\%) & 31,9 & 52,8 & 55,3 & 97,2 \\
MA-PAA (\%) & 23,3 & 55,2 & 56,5 & 100,0 \\
HC (Puntos) & 7,0 & 18,0 & 19,7 & 38,0 \\
\hline
\end{tabular}

En la tabla 2, aparecen las correlaciones de los cursos iniciales de las carreras en estudio con нс, PAA y la parte de Matemática de la PAA. Se decidió incorporar la parte de Comprensión de
Lectura de la PAA (CL-PAA) en el estudio de correlaciones, debido a que se descubrió que Física Introductoria (Fs-0227) correlaciona significativamente $^{1}$ con PAA no por su sección Matemática, sino por su sección de Comprensión de Lectura.

En la tabla 2 se aprecia que нс correlaciona con todos los cursos introductorios de manera positiva, además esta correlación es significativa a un nivel del 5\% con todos los cursos a excepción de Fs-0227. La PAA y ninguna de sus partes presenta una correlación significativa con los cursos introductorios de Estadística y de Matemática, pero sí para Física, donde el componente de comprensión de lectura (cLPAA) sí presenta una correlación significativa.

TABLA 2

CORRELACIONES ENTRE LAS PRUEBAS Y LOS CURSOS INTRODUCTORIOS

\begin{tabular}{lllllllllll}
\hline \multirow{2}{*}{ CARRERA } & \multirow{2}{*}{ CURSOS } & \multicolumn{3}{c}{ HC } & \multicolumn{2}{c}{ PAA } & \multicolumn{2}{c}{ MA-PAA } & \multicolumn{2}{c}{ CL-PAA } \\
\cline { 3 - 11 } & & $\mathrm{r}$ & $\mathrm{p}$ & $\mathrm{r}$ & $\mathrm{p}$ & $\mathrm{r}$ & $\mathrm{p}$ & $\mathrm{r}$ & $\mathrm{p}$ \\
\hline Física & FS-0227 & 0,380 & 0,061 & $0,454^{*}$ & 0,039 & 0,305 & 0,178 & $0,759^{*}$ & 0,000 \\
Física & MA-1004 & $0,436^{*}$ & 0,013 & $0,413^{*}$ & 0,032 & $0,392^{*}$ & 0,043 & $0,479^{*}$ & 0,012 \\
Estadística & XS-1110 & $0,368^{*}$ & 0,005 & 0,199 & 0,200 & 0,244 & 0,115 & 0,114 & 0,465 \\
Matemática & MA-0150 & $0,442^{*}$ & 0,021 & 0,260 & 0,200 & 0,233 & 0,252 & 0,158 & 0,441 \\
\hline
\end{tabular}

r: valor de la correlación p: significancia estadística

En la tabla 3 se observa el alto nivel de deserción que hay en estas carreras, por ejemplo, de 90 estudiantes admitidos en las carreras de Física y Meteorología, el 72,22\% no llevaron el curso de Fs-0227 o se trasladaron de carrera. Solo en Estadística, el porcentaje de deserción es menor al $40 \%$. Por otro lado, se puede ver un grave problema en los índices de aprobación en ma-0150 y Fs-0227, ya que ni siquiera un 50\% de los estudiantes que deciden llevarlo, logran aprobarlo.
TABLA 3

FRECUENCIAS BASADAS EN LOS CURSOS INTRODUCTORIOS

\begin{tabular}{lccc}
\hline & FÍSICA & ESTADÍSTICA & MATEMÁTICA \\
\hline Aprobados & 10 & 51 & 4 \\
Reprobados & 15 & 5 & 23 \\
Desertores & 65 & 9 & 24 \\
\hline total & 90 & 65 & 51 \\
\hline
\end{tabular}


La tabla 4 presenta la información que respecta a los grupos formados con base en la aprobación de los cursos de tercer ciclo de carrera. Se aprecia que en Estadística, la carrera que tenía índices más favorables con respecto a su curso introductorio, presenta un rezago de casi el $75 \%$ entre los estudiantes que matricularon Estadística Introductoria. En Matemática y Física, el rezago es similar a lo observado con respecto al rendimiento en el curso introductorio.

TABLA 4

FRECUENCIAS BASADAS EN LOS CURSOS DE TERCER CICLO

\begin{tabular}{lccc}
\hline & FÍSICA & ESTADÍSTICA & MATEMÁTICA \\
\hline Avanzados & 8 & 15 & 5 \\
Rezagados & 17 & 41 & 22 \\
Desertores & 65 & 9 & 24 \\
\hline total & 90 & 65 & 51 \\
\hline
\end{tabular}

Al realizar el Anova basado en FS-0227, se obtuvieron evidencias de que al menos uno de los promedios de HC, PAA (MA-PAA $y$ CL-PAA) era distinto, a un nivel de significancia de $5 \%$. Según la prueba de comparaciones múltiples de Tuckey que se presenta en la tabla 5, estas diferencias son significativas entre los reprobados y desertores, asimismo, entre los aprobados y los desertores, para las puntuaciones obtenidas en hc, PAa $y$ ma-PaA. Solo en CL-PaA se observaron diferencias significativas entre los aprobados con los desertores y reprobados. En todos los casos, las diferencias son el sentido esperado, esto es, los aprobados tienen mejores promedios que los reprobados o los desertores, $y$ los reprobados tienen mejores promedios que los desertores.

TABLA 5

COMPARACIONES DE TUCKEY PARA LAS CARRERAS DE FÍSICA SEGÚN EL CURSO FS-0227

\begin{tabular}{lccccccccc}
\hline \multirow{2}{*}{ Grupos } & \multicolumn{3}{c}{ HC } & \multicolumn{2}{c}{ PAA } & \multicolumn{2}{c}{ MA-PAA } & \multicolumn{3}{c}{ CL-PAA } \\
\cline { 2 - 10 } & dif & p & dif & p & dif & p & dif & p \\
\hline Reprobados-Desertores & $6,723^{*}$ & 0,000 & $16,730^{*}$ & 0,000 & $21,949 *$ & 0,000 & 6,977 & 0,243 \\
Aprobados-Desertores & $9,823^{*}$ & 0,000 & $26,862^{*}$ & 0,000 & $28,693^{*}$ & 0,000 & $25,649^{*}$ & 0,000 \\
Aprobados-Reprobados & 3,100 & 0,336 & 10,132 & 0,098 & 6,743 & 0,564 & $18,672^{*}$ & 0,007 \\
\hline
\end{tabular}

dif: diferencia entre promedio.

De manera análoga en los Anova basados en ма-1004, se encontraron diferencias significativas entre los promedios. Entre los resultados de las comparaciones múltiples presentados en la tabla 6 , destaca que únicamente en нс hay diferencias significativas en las tres parejas de comparaciones, las cuales se dieron en la dirección esperada.

TABLA 6

COMPARACIONES DE TUCKEY PARA LAS CARRERAS DE FÍSICA SEGÚN EL CURSO MA-1004

\begin{tabular}{lccccccccc}
\hline \multirow{2}{*}{ GRUPOS } & \multicolumn{2}{c}{ HC } & \multicolumn{2}{c}{ PAA } & \multicolumn{2}{c}{ MA-PAA } & \multicolumn{3}{c}{ CL-PAA } \\
\cline { 2 - 10 } & dif & $\mathrm{p}$ & dif & $\mathrm{p}$ & dif & $\mathrm{p}$ & dif & $\mathrm{p}$ \\
\hline Reprobados-Desertores & $3,632^{*}$ & 0,030 & $9,840^{*}$ & 0,016 & $12,231^{*}$ & 0,021 & 5,667 & 0,313 \\
Aprobados-Desertores & $9,966^{*}$ & 0,000 & $20,751^{*}$ & 0,001 & $25,076^{*}$ & 0,001 & $21,460^{*}$ & 0,001 \\
Aprobados-Reprobados & $6,333^{*}$ & 0,023 & 10,911 & 0,149 & 12,845 & 0,199 & $15,793^{*}$ & 0,042 \\
\hline
\end{tabular}


Para concluir con respecto a las carreras de Física, se observa en la tabla 7 , que las cuatro puntuaciones presentan diferencias significativas entre los rezagados $y$ desertores, así como, entre los avanzados $y$ los desertores, ambas en contra de los desertores. Por otro lado, en ninguna prueba se presentan diferencias significativas entre los promedios de los avanzados y los rezagados.

TABLA 7

COMPARACIONES DE TUCKEY PARA LAS CARRERAS DE FÍSICA SEGÚN LOS CURSOS DE TERCER CICLO DE CARRERA

\begin{tabular}{lccccccccc}
\hline \multirow{2}{*}{ Grupos } & \multicolumn{2}{c}{ HC } & \multicolumn{2}{c}{ PAA } & \multicolumn{2}{c}{ MA-PAA } & \multicolumn{2}{c}{ CL-PAA } \\
\cline { 2 - 10 } & dif & $\mathrm{p}$ & dif & $\mathrm{p}$ & dif & $\mathrm{p}$ & dif & $\mathrm{p}$ \\
\hline Rezagados -Desertores & $7,558^{*}$ & 0,000 & $19,781^{*}$ & 0,000 & $24,046^{*}$ & 0,000 & $11,805^{*}$ & 0,018 \\
Avanzados -Desertores & $8,823^{*}$ & 0,000 & $23,654^{*}$ & 0,000 & $26,427^{*}$ & 0,000 & $21,328^{*}$ & 0,001 \\
Avanzados- Rezagados & 1,265 & 0,849 & 3,873 & 0,738 & 2,381 & 0,937 & 9,524 & 0,321 \\
\hline
\end{tabular}

Los resultados de la comparaciones en Estadística basados en el curso introductorio, se presentan en la tabla 8 , mientras que los basados en los cursos de tercer ciclo se presentan en la tabla 9. En estas tablas se aprecia que tanto la PAA como la MA-PAA no presentan diferencias significativas entre ninguno de los pares de grupos definidos para este estudio. A los estudiantes que no llegan ni tan siquiera a cursar xs-1110 les va mejor que a los que lo llevan y lo reprueban la primera vez, lo cual es contrario a lo esperado.

TABLA 8

COMPARACIONES DE TUCKEY PARA ESTADÍSTICA

SEGÚN EL CURSO XS-1110

\begin{tabular}{lccccccc}
\hline \multirow{2}{*}{ Grupos } & \multicolumn{3}{c}{ HC } & \multicolumn{2}{c}{ PAA } & \multicolumn{3}{c}{ MA-PAA } \\
\cline { 2 - 8 } & dif & $\mathrm{p}$ & dif & $\mathrm{p}$ & dif & $\mathrm{p}$ \\
\hline Reprobados-Desertores & 2,222 & 0,731 & $-6,373$ & 0,623 & $-9,833$ & 0,571 \\
Aprobados-Desertores & $5,418^{*}$ & 0,016 & 3,512 & 0,733 & 8,869 & 0,377 \\
Aprobados-Reprobados & 3,196 & 0,404 & 9,885 & 0,190 & 18,703 & 0,056 \\
\hline
\end{tabular}

Los promedios obtenidos en Hc reflejan diferencias significativas en el sentido esperado entre los que aprueban xs-1110 y los que no lo matriculan, y entre los que han aprobado algún curso de tercer ciclo y los que dejaron la carrera.
Además, se presentan diferencias significativas a un nivel del $10 \%$ en los promedios de $\mathrm{Hc}$, al comparar los estudiantes rezagados con los desertores y al comparar los estudiantes avanzados con los rezagados. 
TABLA 9

COMPARACIONES DE TUCKEY PARA ESTADÍSTICA

SEGÚN LOS CURSOS DE TERCER CICLO DE CARRERA

\begin{tabular}{lccccccc}
\hline \multirow{2}{*}{ Grupos } & \multicolumn{2}{c}{ HC } & \multicolumn{3}{c}{ PAA } & \multicolumn{3}{c}{ MA-PAA } \\
\cline { 2 - 8 } & dif & p & dif & p & dif & p \\
\hline Rezagados -Desertores & 3,997 & 0,081 & 0,936 & 0,978 & 5,171 & 0,737 \\
Avanzados -Desertores & $7,356^{*}$ & 0,002 & 7,801 & 0,336 & 13,223 & 0,237 \\
Avanzados- Rezagados & 3,358 & 0,073 & 6,866 & 0,185 & 8,052 & 0,335 \\
\hline
\end{tabular}

Finalmente, las comparaciones para las carreras de Matemática basadas en el curso introductorio $y$ en los cursos de tercer ciclo se presentan en las tablas 10 y 11, respectivamente. Al igual que para Estadística, no se presentan diferencias significativas en los promedios obtenidos en PAA $y$ en MA-PAA, además, contrario a lo esperado, se presentan diferencias observadas a favor de los desertores al compararlos con los que reprueban ма-0150 y los estudiantes rezagados.

TABLA 10

COMPARACIONES DE TUCKEY PARA LAS CARRERAS DE MATEMÁTICA SEGÚN EL CURSO MA-0150

\begin{tabular}{lccccccc}
\hline \multirow{2}{*}{ Grupos } & \multicolumn{2}{c}{ HC } & \multicolumn{2}{c}{ PAA } & \multicolumn{3}{c}{ MA-PAA } \\
\cline { 2 - 8 } & dif & $\mathrm{p}$ & dif & $\mathrm{p}$ & dif & $\mathrm{p}$ \\
\hline Reprobados-Desertores & 1,264 & 0,756 & $-1,739$ & 0,890 & $-3,903$ & 0,716 \\
Aprobados-Desertores & $11,667^{*}$ & 0,002 & 11,832 & 0,213 & 14,413 & 0,265 \\
Aprobados-Reprobados & $10,402^{*}$ & 0,007 & 13,572 & 0,137 & 18,316 & 0,125 \\
\hline
\end{tabular}

Los puntajes obtenidos en Hc revelan diferencias significativas a favor de los que aprueban ма- 0150 con respecto a los desertores y los que reprueban el curso, también muestra diferencias significativas a favor de los que avanzan en la carrera con respecto a los que quedan rezagados y a los que desertaron.

TABLA 11

COMPARACIONES DE TUCKEY PARA LAS CARRERAS DE MATEMÁTICA SEGÚN LOS CURSOS DE TERCER CICLO DE CARRERA

\begin{tabular}{lccccccc}
\hline \multirow{2}{*}{ Grupos } & \multicolumn{2}{c}{ HC } & \multicolumn{3}{c}{ PAA } & \multicolumn{3}{c}{ MA-PAA } \\
\cline { 2 - 7 } & dif & $\mathrm{p}$ & dif & $\mathrm{p}$ & dif & $\mathrm{p}$ \\
\hline Rezagados -Desertores & 1,053 & 0,827 & $-1,991$ & 0,863 & $-4,304$ & 0,673 \\
Avanzados -Desertores & $10,517^{*}$ & 0,003 & 10,175 & 0,251 & 12,436 & 0,302 \\
Avanzados- Rezagados & $9,464^{*}$ & 0,008 & 12,166 & 0,149 & 16,741 & 0,126 \\
\hline
\end{tabular}




\section{DISCUSIÓN}

El estudio sobre la comparación de los promedios de la Prueba de Aptitud Académica y la Prueba de Habilidades Cuantitativas de los estudiantes de las carreras de Física, Estadística y Matemática realizado en esta investigación, ha permitido constatar que una cantidad considerable de estudiantes ingresa a estas carreras con poco interés en estas áreas del conocimiento, lo cual se aprecia en el alto índice de deserción que se da en los cursos introductorios de las carreras de Física y Matemática, principalmente.

Por otro lado, se ha verificado que también ingresan estudiantes que no consideran previamente las habilidades y características deseables, indicadas en las fichas profesiográficas $y$ que son necesarias para poder desempeñarse con éxito, lo cual se percibe notablemente en la cantidad de estudiantes reprobados y rezagados para todas las carreras que participan de este estudio, en particular, para Estadística, que si bien no presentó altos niveles de deserción en los cursos introductorios, la cantidad de rezagados presentó un aumento considerable.

Los análisis reportados en este informe revelan que la prueba de Habilidades Cuantitativas (нс) de la uсR, que se ha venido aplicando desde el 2008 sin ser vinculante, ofrece resultados significativos cuando se establecen las correlaciones entre las pruebas (PAA, MA-PAA $y$ CL-PAA) y нс, para la categoría de cursos introductorios de las carreras analizadas; es precisamente esta última prueba la que aporta resultados relevantes para casi todos estos cursos, no así la paA y sus partes, que correlacionan únicamente con los cursos introductorios de las carreras de Física.

En las comparaciones realizadas para cada uno de los grupos incluidos en la categoría de cursos introductorios pertenecientes a las carreras de Física y específicamente, para el curso Fs-0227, las cuatro pruebas ofrecen diferencias significativas en cuanto a que los aprobados obtienen mejores promedios que los desertores. Según Hc, PAA $y$ MA-PAA a los reprobados les va mejor que a los que deciden abandonar la carrera, no así para CL-PAA que no aportó resultados significativos. Sin embargo, cuando se analiza el curso ма-1004, es notorio que la única prueba que correlaciona de manera positiva con todos los grupos es la Hc, brindando resultados que confirman que los estudiantes aprobados obtienen mejores notas que los desertores $y$ reprobados, y a estos a su vez les va mejor que los que deciden abandonar la carrera.

Para la categoría de los cursos del tercer ciclo, las cuatro pruebas arrojan diferencias significativas con respecto a los grupos de avanzados-desertores $y$ rezagados-desertores, $y$ de nuevo se verifica que a los estudiantes que se mantienen en la carrera les va mejor comparados con los que la abandonan. No así con respecto al grupo de los avanzados-rezagados, en la cual ninguna de las pruebas aportó diferencias relevantes. En resumen, para las carreras de Física, el test de нс ofrece resultados significativos para el curso introductorio ма-1004, en todos los grupos definidos. No así para el curso FS- 0227 y los del tercer ciclo, donde los resultados ofrecidos son similares a los obtenidos por la PAA y sus componentes.

Con respecto a la carrera de Estadística $y$ en ambas categorías, el curso introductorio $y$ de tercer ciclo, la única prueba que presenta diferencias significativas en cuanto al grupo de los estudiantes que aprueban-desertan y entre los que avanzan-desertan, es la нc, logrando también mayores promedios los que aprueban y avanzan en comparación con los que desertan; lo que evidencia que la prueba de $\mathrm{Hc}$ puede detectar a aquellos estudiantes que tienen las habilidades mínimas para cursar esta carrera. Pero los resultados arrojados por las pruebas de PAA y мA-PAa en la categoría de curso introductorio, son un llamado de alerta para la carrera y la universidad misma, pues muestran a los desertores con mejores promedios que los reprobados, lo que genera un gasto considerable de recursos por parte de la Universidad, al admitir estudiantes que no poseen las competencias mínimas.

Otro resultado importante se da para las carreras de Matemática y en ambas categorías, nuevamente, la prueba de $\mathrm{HC}$ es la que ofrece diferencias significativas. En el curso introductorio, entre los que aprueban-desertan y los que aprueban-reprueban, obtienen mejor puntuación los aprobados. Algo similar ocurre en 
la categoría de cursos de tercer ciclo, donde se aprecia que en los grupos de avanzados-desertores y avanzados-rezagados, los avanzados obtienen mejores resultados sobre los demás. Los promedios obtenidos en las pruebas de PAA $y$ мАPAA para ambas categorías, vuelven a revelar que a los desertores les va mejor que a los reprobados y rezagados, situación preocupante y que al igual que para Estadística, debe hacer reflexionar acerca de la necesidad de utilizar un test como el de нс, el cual está demostrando tener un poder predictivo eficiente, principalmente para estas últimas carreras; al igual que lo hace la sección de habilidades cuantitativas del exнсоBA de la Universidad Autónoma de Baja California y el GRE Avanzado Prueba de Matemática.

La utilización de las pruebas específicas y en particular, las pruebas que miden habilidades cuantitativas, es una realidad; el empleo que hoy se hace de ellas en diferentes contextos educativos principalmente, a nivel superior, además de los estudios que se realizan para comprobar su validez predictiva, deja patente la necesidad que hay dentro de estas instituciones por mejorar sus procesos de selección, de manera tal que facilite la identificación de aquellos aspirantes que tienen mayores posibilidades de cursar y terminar una carrera. Esto fortalece lo expresado por Thurstone, L. y Thurstone, T. (1941) y Guilford (1967), pues para ellos es mejor medir cada factor que compone la inteligencia por separado, para obtener un perfil de las habilidades de una persona.

Lo anterior cobra relevancia para las universidades estatales, cuya responsabilidad es optimizar al máximo sus recursos, lo cual las compromete a garantizar que los estudiantes quienes ingresan a las diferentes carreras posean las habilidades mínimas deseables o bien, brindar el apoyo oportuno y necesario a aquellos estudiantes que así lo ameriten para aumentar sus posibilidades de éxito como lo concluyen Schneider y Briel (1990). Esto traerá réditos a las propias universidades, pues estarían asegurándose un buen nivel académico por parte de sus estudiantes desde el inicio de los estudios superiores e incrementarían las probabilidades de que estos concluyan los mismos.

\section{BIBLIOGRAFÍA}

LIBROS

Carrol, J. B. Human cognitive abilities. usa: Cambride University Press, 1993.

De Juan-Espinoza, M. Geografía de la inteligencia humana. España: Pirámide, 1997.

Escrigas, C. y Lobera, J. "Nuevas dinámicas para la responsabilidad social". La educación superior en tiempos de cambio. Global University Network for Innovation (ed.). Madrid, España. Ediciones Mundi-Prensa, 2009: 3-16.

González Tirados, R. M. Análisis de las causas del fracaso escolar en la Universidad Politécnica de Madrid. Madrid: MEc-CIDE, 1989.

González Tirados, R.M. "Principales dificultades en el rendimiento académico en primer año de carreras de ingeniería". La investigación educativa sobre la universidad. Aurora Blanco Mariclla, Mercedes Muñoz-Repiso Izaguirre, Bienvenida Margarita Latiesa Rodríguez, Rosa María González Tirados (eds.). Madrid: Centro de Publicaciones del Ministerio de Educación y Ciencia (CIDE), 1990.

González Tirados, R.M. Rendimiento académico en la Universidad Politécnica de Madrid. Estudio longitudinal en primer ciclo 1989-93. Tomos I y II. Madrid: Servicio de Publicaciones de la Universidad Politécnica/ıce, 1993.

Guilford, J.P. The nature of human intelligence. Nueva York: McGraw-Hill, 1967.

National Council of Teachers of Mathematics. Principles and standards for school mathematics. Reston, va: National Council of Teachers of Mathematics, 2000.

Page, M.; Moreal, B.; Calleja, J.A.; Cerdan, J.; Echevarria, M.J.; Garcia, C.; Garivia, J.L.; Gómez, C.; Jiménez, S.C.; López, B.; Martín-Javato, L.; Mínguez, A.L.; Sánchez, A. y Trillo, C. Hacia un modelo causal del rendimiento académico. Madrid: España, 1990. 
Pérez, R. La Universidad Nacional de Educación a Distancia. Aproximación a la evaluación de un modelo innovador. Madrid: Centro de Publicaciones del Ministerio de Educación y Ciencia (CIDE), 1991.

Pérez-Luño, A.; Ramón Jerónimo, J. y Sánchez, V. J. Análisis exploratorio de las variables que condicionan el rendimiento académico. Sevilla, España. Universidad Pablo de Olavide, 2000.

Proyecto de Habilidades Cuantitativas. Construcción y validación de un banco de items y pruebas de habilidades cuantitativas para diagnóstico e ingreso a carrera en la Universidad de Costa Rica. Documento oficial del Proyecto. 2010.

Rojas, L. Validez predictiva de los componentes del promedio de admisión a la Universidad de Costa Rica. Manuscrito inédito. Instituto de Investigaciones PsicológicasUniversidad de Costa Rica, 2012.

Steen, L. A. Achieving quantitative literacy: an urgent challenge for higher education. Washington, DC: Mathematical Association of America, 2004.

Thurstone, L. L. y Thurstone, T. G. "Factorial studies of intelligence". Psychometric Monographs 2. 1941.

Tourón, F. J. Factores del rendimiento académico en la Universidad. Pamplona: Ediciones Universidad de Navarra, 1984.

REVISTAS

Boza, G. "Rendimiento académico y satisfacción estudiantil: una evaluación del sistema universitario mexicano". Revista Mexicana de Sociología 32 (4). Memorias del ix Congreso Latinoamericano de Sociología, 1970: 1005-1042.

De Spinola, H. "Rendimiento académico y factores psicosociales en los ingresantes a la carrera de medicina - unNE". Revista Paraguaya de Sociología 78. 1990:143167.

Garbanzo, V. G. "Factores asociados al rendimiento académico en estudiantes universitarios, una reflexión desde la calidad de la educación superior pública". Revista Educación 31 (1). 2007: 43-63.

Jiménez, H. M. "Competencia Social: intervención preventiva en la escuela". Infancia y Sociedad 24. 1994.

Jiménez, K. y Morales, E. "Validez predictiva del Promedio de Admisión de la Universidad de Costa Rica y sus componentes". Actualidades en Psicología 23-24. 20092010: 21-55.

Lobato, J. y Siebert, D. "Quantitative reasoning in a reconceived view of transfer". Journal of Mathematical Behavior 21. 2002: 87-116.

Paglin, M. y Rufolo, A. "Heterogeneous Human Capital, Occupational Choice, and Male-Female Earnings Differences". Journal of Labor Economics 8 (1). The University of Chicago Press on behalf of the Society of Labor Economists and the NORC at the University of Chicago Stable, 1990: 123-144.

Rusell, J.; Persing, D.; Dunn, J. y Rankin, R. "Focusing on the selection process: a field study of the measures that influence admission decisions". Educational and Psochological Measurement 50 (4). 1990: 901-913.

Thompson, P. "Quantitative reasoning, complexity an additive structures". Educational Studies in Mathematics 25 (3). 1993: 165-208.

Tirado, F.; Backhoff, E.; Larrazolo, N. y Rosas, M. "Validez predictiva del Examen de Habilidades y Conocimientos Básicos (ехнсова)". Revista Mexicana de Investigación Educativa 2 (3). Junio 1997: 67-84.

Vélez Van, M. A. y Roa, N. C. "Factors associated with academic perfomance in medical students". psIc. Educación Médica 2 (8). 2005: 1-10.

\section{TEXTOS ELECTRÓNICOS}

Cascón, V. I. Predictores del rendimiento académico en alumnos de primero $y$ segundo de BUP. 2000. En: <http:// campus.usal.es/ inico/investigacion/ 
jornadas/jornada $2 /$ comun/c19.html> [consultado el 21 de mayo de 2012].

Dwyer, C.; Gallagher, A.; Levin, J. y Morley, M. What is quantitative reasoning? Defining the construct for assessment purposes. 2003. En: <http://www.ets.org/Media/ Research/pdf/RR-03-30-Dwyer.pdf> [consultado el 5 de junio de 2012].

Edel, R. "El rendimiento académico: concepto, investigación y desarrollo". Revista Electrónica Iberoamericana sobre Calidad, Eficacia y Cambio en Educación 1 (2). 2003. En: <http://www. ice.deusto.es/rinace/reice/volln2/Edel. pdf $>$ [consultado el 21 de mayo de 2012].

Karim, N. Quantitative reasoning applications and modelling in the real world. Zayed University, s.f. En: <http://math.unipa. it/ grim/21_project/21_Charlotte_ KarimPaperEdit.pdf> [consultado el 22 de mayo de 2012].

Montero, E.; Villalobos, J. y Valverde, A. "Factores institucionales, pedagógicos, psicosociales y sociodemográficos asociados al rendimiento académico en la Universidad de Costa Rica: un análisis multinivel". Revista Relieve 13. 2007. En: $<$ http://www.uv.es/RELIEVE/v13n2/RELIEVEv 13n2_5.htm> [consultado el 7 de mayo de 2012].

Programa Estado de la Nación. Tercer Informe Estado de la Educación. San José: Programa Estado de la Nación, 2011.
En: <http://www.estadonacion.or.cr/ index.php/biblioteca-virtual/costaica/educacion /sinopsis/informe-iii> [consultado el 12 de marzo de 2012].

Schneider, L. y Briel J. "Validity of the GRE: 1988-89 Summary Report". Educational Testing Service. 1990. En: <http://www. ets.org/Media/Research/pdf/greв-90 1vss.pdf> [consultado el 31 de agosto de 2012].

The Graduate Record Examinations. "The prediction of doctorate attainment in Psychology, Mathematics and Chemistry". Report bREB No. 69-6aR. New Jersey: Educational Testing Service Princeton, 1972. En: <http://www.ets. org/Media/Research/pdf/Greb-69-06ar. pdf $>$ [consultado el 14 de setiembre de 2012].

Universidad de Costa Rica. Oficina de Orientación. 2010. En: <http://www. orientacion. ucr.ac.cr/?q=node/143> [consultado el 14 de marzo de 2012].

Universidad de Costa Rica. Oficina de Planificación Universitaria. 2012. En: <http://oplau.UCR.ac.cr /index.php/ publicaciones/panorama-cuantitativouniversitario $>$ [consultado el 6 de junio de 2012].

Fecha de ingreso: 29/11/2012 Fecha de aprobación: 26/04/2013 
